\title{
Thermodynamics of charged accelerating AdS black holes and holographic heat engines
}

\section{Jialin Zhang, Yanjun Li and Hongwei Yu}

Department of Physics and Synergetic Innovation Center for Quantum Effects and Applications, Hunan Normal University, Changsha, Hunan 410081, China

E-mail: jialinzhang@hunnu.edu.cn, 583048768@qq.com, hwyu@hunnu.edu.cn

ABSTRACT: Using a reasonable choice in normalizing the timelike Killing vector, we investigate the thermodynamic properties of charged accelerating Anti-de Sitter (AdS) black holes. We find that the expression of the thermodynamic mass in the first law of thermodynamics displays an inextricably intertwining behavior with the charge due to the unusual asymptotic structure of the accelerating black holes. Meanwhile, the thermodynamic length as a potential conjugate to the varying cosmic string tension is introduced and analyzed in detail, and the possible phase behavior of the charged accelerating black holes is also discussed in a standard thermodynamic analysis. Furthermore, we also investigate the properties of holographic heat engines with charged accelerating AdS black holes as the working substance in a benchmarking scheme. We find that the efficiencies of the black hole heat engines can be influenced by both the size of the benchmark circular cycle and the cosmic string tension. More interestingly, the existence of charge may significantly increase the efficiencies of the black hole heat engines and make them be more sensitive to a varying cosmic string tension. A cross-comparison of the efficiencies of different black hole heat engines suggests that the acceleration also increases the efficiency and renders it more sensitive as the charge varies.

KeYwords: AdS-CFT Correspondence, Black Holes

ARXIV ePrint: 1808.10299 


\section{Contents}

1 Introduction 1

2 The charged accelerating AdS black hole and its thermodynamics 3

$\begin{array}{lll}3 & \text { Benchmarking black hole heat engines and efficiency } & 7\end{array}$

4 Conclusion $\quad 12$

\section{Introduction}

Ever since Hawking's pioneering work on the radiation of black holes [1, 2], the black hole thermodynamics has become an important topic of intensive research and been considered as a key to gaining insight into the quantum nature of gravity. In the last decades, the thermodynamics of several classes of black holes has been studied, and remarkable progresses have been made in, for example, connecting the laws of gravitation with the laws of thermodynamics [3, 4], deploying the black holes as a holographically dual system in quantum chromodynamics [5] and condensed matter physics [6, 7], and approaching the thermodynamics of black holes geometrically [8-17].

Recent studies show that the thermodynamical properties of black holes in anti-de Sitter (AdS) space are quite different from those in flat or de Sitter(dS) space. In particular, a subject dubbed black hole chemistry $[18,19]$, in which the negative cosmologic constant $\Lambda$ is regarded as a thermodynamic pressure in the extended phase space [20-26], has started to attract a growing deal of interest. To date, the investigation in black hole chemistry has led to the discovery of a lot of interesting thermodynamic phenomena, such as the analogous phase of van der Waals fluid [27], re-entrant phase transitions [28] and the analogous superfluid phase transitions [29].

In the context of black hole chemistry, the concept of holographic heat engines which can extract work with the AdS black holes as the working substance in cycles in the pressure-volume phase plane was proposed by Johnson [30]. Subsequently, in order to better crossly compare the efficiency of the heat engines with different black holes as the working material, a circular cycle of the heat engine in the pressure-volume plane has been introduced as a benchmarking prescription by Chakraborty and Johnson [31]. After Johnson's pioneering work, the concept of heat engines has been generalized to other different black holes (for an incomplete list of references, see [32-47]). Particularly, the properties of the holographic heat engine with accelerating AdS black holes as the working substance have drawn considerable interest. The accelerating AdS black holes are described by the so-called $C$-metric [48-51], which can be used to study either one or two accelerating black 
holes, the pair creation of black holes [52], the construction of the black ring solution in 5-dimensional gravity [53], and the test of gravitational radiation [54].

However, the $C$-metric, despite of the aforementioned various applications, remains somewhat esoteric in its thermodynamic prescription partly due to the fact that it has an unusual asymptotic behavior and at least one non-removable conical singularity on the azimuthal axis of symmetry. As a result, discrepant and conflicting results have been obtained in the investigation of the thermodynamics of the accelerating AdS black holes [55-59]. For example, a set of thermodynamic variables was obtained in refs. [55-57], where the thermodynamic mass and temperature are associated with the coordinate time rather than with the normalized timelike Killing vector. However, the corresponding first law of thermodynamics was later found to be incorrect [60].

Let us note that alternate expressions for the thermodynamic mass has also been posited by assuming the integrability condition in ref. [58]. It has been argued that for regular accelerating black holes, the acceleration is provided by an external electromagnetic field rather than the conical deficit associated with a string, while for the irregular $C$-metric with conical singularities, the black hole is again accelerated by a pulling string. However, in latter case, the cosmic string tension, which should be considered as a thermodynamic parameter relevant to the varying conical deficit, was not included in the first law.

Recently, A. Anabalon et al. re-examined the thermodynamics of uncharged accelerating AdS black holes by means of the mass and temperature generated by a normalized time that just corresponds to the "time" of an asymptotic observer [59]. Such generated thermodynamic mass has been verified to be consistent with the mass obtained by holographic computing, and hence it reconciles discrepancies and conflicts in the previous investigations and confirms at this point that such choice of normalization of timelike Killing vector is applicable and well grounded. Based on the correct thermodynamics of uncharged accelerating AdS black holes stated in ref. [59], the properties of the corresponding benchmarking holographic heat engines have been studied in detail [46]. However, a formulation of the first law of thermodynamics and an examination of the holographic heat engines when the accelerating black hole is charged, where the additional special asymptotic structure of the gauge field renders the thermodynamics a non-trivial task, are quite challenging [59]. More recently, A. Anabalon et al. pointed out in ref. [61] that the choice of normalizing timelike Killing vector can be appropriately fixed by holographic methods for charged accelerating black holes, which takes into account of that all the thermodynamic parameters are analogous to those of an observer who is comoving with an accelerating charged object and does not see any radiation. The purpose of the present paper is to explore the consistent thermodynamic properties for the charged accelerating AdS black holes and study the influence of charge on the corresponding holographic heat engines.

This paper is organized as follows. We begin in section 2 by exploring the explicit expressions for the thermodynamic parameters of charged accelerating AdS black holes with an appropriate normalized time. In section 3, we investigate the properties of the benchmarking holographic heat engines with charged accelerated AdS black holes as working substances. We end with a summary and a conclusion in section 4 . 


\section{The charged accelerating AdS black hole and its thermodynamics}

A charged accelerating AdS black hole is described by the following $C$-metric $[51,55,56]$

$$
d s^{2}=\frac{1}{\Omega^{2}}\left[f(r) d t^{2}-\frac{d r^{2}}{f(r)}-r^{2}\left(\frac{d \theta^{2}}{g(\theta)}+g(\theta) \sin ^{2} \theta \frac{d \phi^{2}}{K^{2}}\right)\right],
$$

where

$$
\begin{aligned}
\Omega & =1+A r \cos \theta, \\
f(r) & =\left(1-A^{2} r^{2}\right)\left(1-\frac{2 m}{r}+\frac{e^{2}}{r^{2}}\right)+\frac{r^{2}}{\ell^{2}}, \\
g(\theta) & =1+2 m A \cos \theta+e^{2} A^{2} \cos ^{2} \theta .
\end{aligned}
$$

The corresponding gauge potential can be considered to be [61]

$$
B=-\frac{e}{r} d t+\frac{e}{r_{+}} d t
$$

with $r_{+}$representing the black hole event horizon. It is easy to deduce that this gauge potential vanishes at the horizon. Here, the parameters $m, e$ and $A$ are related to the mass, the electric charge and the magnitude of acceleration of the black hole respectively, $\ell$ represents the AdS radius, and $K$ characterizes the conical deficit of the spacetime. Specifically, the conical deficits on the spatial two poles, $\theta_{+}=0$ (the north pole ) and $\theta_{-}=\pi$ (the south pole ) are

$$
\delta_{ \pm}=2 \pi\left[1-\frac{g\left(\theta_{ \pm}\right)}{K}\right] .
$$

Such conical deficit corresponds to the tension of the cosmic string $[55,56]$

$$
\mu_{ \pm}=\frac{\delta_{ \pm}}{8 \pi}=\frac{1}{4}-\frac{1 \pm 2 m A+e^{2} A^{2}}{4 K} .
$$

In order to avoid the occurrence of negative tension defects, clearly we should require $0 \leq \mu_{+} \leq \mu_{-} \leq 1 / 4$.

To obtain the correct thermodynamics, we should first select an appropriate normalized time [62]. As far as metric (2.1) is concerned, such a normalization of the time coordinate can be chosen such that it corresponds to the "time" of an observer comoving with the charged accelerating black hole [61], i.e., $\tau=\alpha t$ with $\alpha=\sqrt{\left(1+A^{2} e^{2}\right)\left(1-A^{2} \ell^{2}-A^{4} \ell^{2} e^{2}\right)}$. In following discussions, we will try to formulate the exact expressions of the thermodynamic parameters for charged accelerating black holes in the frame of normalized time $\tau$. Now the thermodynamic mass can be directly obtained via the conformal methods [63, 64]

$$
M=\left[1-\left(1+A^{2} e^{2}\right) A^{2} \ell^{2}\right] \frac{m}{K \alpha},
$$

which is completely identified with the holographic mass [61].

The temperature $T$ can be found by using the conventional Euclidean method associated with the normalized time,

$$
T=\frac{f^{\prime}\left(r_{+}\right)}{4 \alpha \pi}=\frac{m}{2 \pi \alpha r_{+}^{2}}-\frac{e^{2}}{2 \pi \alpha r_{+}^{3}}+\frac{A^{2} m}{2 \alpha \pi}-\frac{A^{2} r_{+}}{2 \alpha \pi}+\frac{r_{+}}{2 \pi \alpha \ell^{2}}
$$


Here, note that in order to have a well-defined temperature associated with the horizon of the black hole, we assume that the acceleration is small such that the acceleration horizon can be negated by a negative cosmological constant and only the black hole horizon exists [56].

As usual, the entropy $S$ of the accelerating black hole is one quarter of the horizon area

$$
S=\frac{\pi r_{+}^{2}}{K\left(1-A^{2} r_{+}^{2}\right)} .
$$

Meanwhile, the thermodynamic electric charge $Q$ of the black hole can be obtained by

$$
Q=\frac{1}{4 \pi} \int_{\Omega=0} * d B=\frac{e}{K},
$$

with the thermodynamic electrostatic potential

$$
\Phi=\frac{e}{\alpha r_{+}}
$$

and the thermodynamic pressure in extended black hole thermodynamics

$$
P=-\frac{\Lambda}{8 \pi}=\frac{3}{8 \pi \ell^{2}} .
$$

We assume that all the relevant thermodynamic variables should obey the extended first law

$$
\delta M=T \delta S+V \delta P+\Phi \delta Q-\lambda_{+} \delta \mu_{+}-\lambda_{-} \delta \mu_{-} .
$$

Then, according to the Smarr relation

$$
M=2 T S-2 P V+Q \Phi,
$$

it is straightforward to verify

$$
\begin{aligned}
V & =\frac{4 \pi}{3 K \alpha}\left[\frac{r_{+}^{3}}{\left(1-A^{2} r_{+}^{2}\right)^{2}}+A^{2} \ell^{4} m+A^{4} e^{2} \ell^{4} m\right], \\
\lambda_{ \pm} & =\frac{1}{\alpha}\left[\frac{r_{+}}{1 \pm A r_{+}}-\frac{m}{1+A^{2} e^{2}} \mp A \ell^{2}\left(1+A^{2} e^{2}\right)\right],
\end{aligned}
$$

where $\lambda_{ \pm}$are introduced as the thermodynamic lengths conjugate to the tensions $\mu_{ \pm}[56,59]$.

For the case of vanishingly-small acceleration (i.e., $A \ell \ll 1$ and $e A \ll 1$ ), some proposed thermodynamic parameters can be approximated as

$$
\begin{aligned}
M & \approx \frac{m}{K}\left[1-\frac{A^{2}}{2}\left(e^{2}+\ell^{2}\right)\right], \\
V & \approx \frac{4 \pi}{3 K}\left[r_{+}^{3}+\frac{A^{2}}{2}\left(2 \ell^{4} m-e^{2} r_{+}^{3}+\ell^{2} r_{+}^{3}+4 r_{+}^{5}\right)\right], \\
\lambda_{ \pm} & \approx r_{+}-m \mp\left(\ell^{2}+r_{+}^{2}\right) A+\frac{A^{2}}{2}\left[e^{2}\left(3 m-r_{+}\right)+2 r_{+}^{3}+\ell^{2}\left(r_{+}-m\right)\right] .
\end{aligned}
$$


In order to gain a better understanding of the thermodynamic behavior, let us recall the expression of the conical deficits in eq. (2.6). As we see that the conical deficits in this spacetime are unequal on different axes, so it is impossible to choose a fixed $K$ to simultaneously regularize the metric at both two poles. Such kind of irregularity along an axis is just precisely a definition of a conical singularity which encodes the information about the presence of cosmic string. Actually, the regularity of the metric at one pole demands

$$
K_{ \pm}=g\left(\theta_{ \pm}\right)=1 \pm 2 m A+e^{2} A^{2}
$$

Here, we choose $K=K_{+}=1+2 m A+e^{2} A^{2}$ so that the metric is just regular on the north pole (i.e., $\mu_{+}=0$ ), leaving a conical deficit on the south pole, then the string tension on the south pole yields $\mu_{-}=m A / K$.

Before further exploring the thermodynamical behaviours of charged accelerating black holes, a few comments concerning the normalization factor $\alpha$ are in order. As stated previously, the assumption of a "slowly accelerating black hole" ensures that the temperature of the black hole is well-defined, the accelerating horizon is removed, and all the thermodynamic variables in the first law are unambiguous. Explicitly, the slowly accelerating $C$-metric means that the parameters of the solution in metric (2.1) satisfy $A \ell<1$ and $2 A m<1[55,56]$. Furthermore, it is straightforward to verify that $e^{2} A^{2}<1$ by considering the presence of the event horizon of the black hole, i.e, using $f\left(r_{+}\right)=0$ and $1>A r_{+}>0$. Thus, we have

$$
\frac{\alpha_{0}}{\alpha}=\frac{\sqrt{1-A^{2} \ell^{2}}}{\sqrt{\left(1+A^{2} e^{2}\right)\left(1-A^{2} \ell^{2}-A^{4} \ell^{2} e^{2}\right)}}=1-\frac{e^{2} A^{2}}{2}+\left(\frac{3 e^{4}}{8}+\frac{e^{2} \ell^{2}}{2}\right) A^{4}+\ldots,
$$

where $\alpha_{0}=\sqrt{1-A^{2} \ell^{2}}$ denotes the normalization factor without charge. As we can see form eq. (2.19), the ratio $\alpha_{0} / \alpha$ approximates to 1 for $A^{2} e^{2} \ll 1$ and $A \ell<1$, and this just is the more slowly accelerating requirement. In addition, it is worth pointing out that, practically, the value of the parameter $e$ (or $Q$ ) can not be chosen arbitrarily large, and it should keep the corresponding charged black hole far away from the extremal case where the temperature vanishes. Therefore, the difference between $\alpha_{0}$ and $\alpha$ usually is tiny if the slowly accelerating assumption $\left(A^{2} e^{2} \ll 1\right.$ and $\left.A \ell<1\right)$ is strictly applied.

Let us return to the thermodynamic variables. We can see, from figure 1 , that when the renormalized thermodynamic mass $M$ is comparable to AdS radius $\ell$, the thermodynamic length $\lambda_{-}$becomes negative. And the thermodynamic length is not always a monotonic decreasing function of $M$ in the region of $M / \ell<1$, and the functional behavior depends on the concrete values of $\mu_{-}$and $Q$. Furthermore, when $M$ becomes very large for a fixed tension $\mu_{-}$, the influence of the charge variation on the thermodynamic length almost disappears. Here, notice that if $M / \ell$ is smaller than that of the extremal black hole, the temperature becomes negative, which is usually considered as unphysical. Moreover, it should be pointed out that as $A \ell$ is drawing near one, the presence of the acceleration horizon makes the thermodynamic temperature ambiguous $[55,56,59]$. So, the yellow curve $\left(\mu_{-}=0.15\right)$ in figure 1 (a) does not continue to arbitrarily small $M / \ell$ (i.e., $M / \ell \gtrsim 0.0232$ or equivalently $A \ell \lesssim 0.9783)$ in order for the thermodynamic temperature of accelerating 


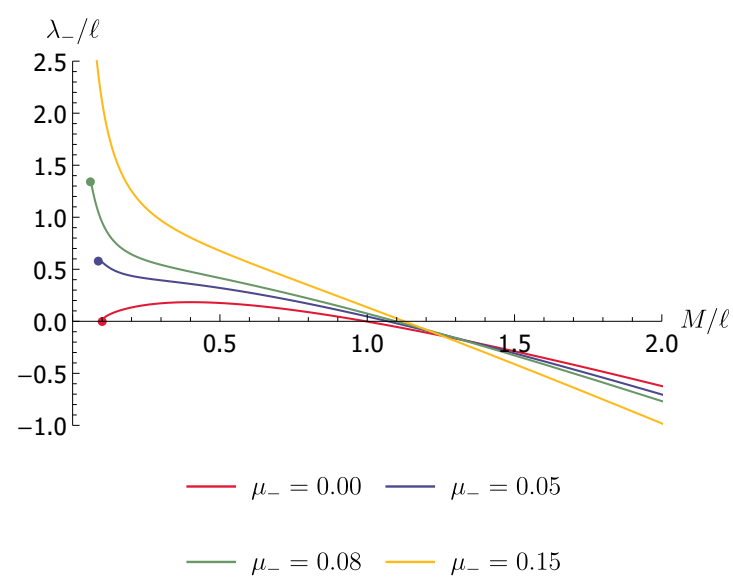

(a)

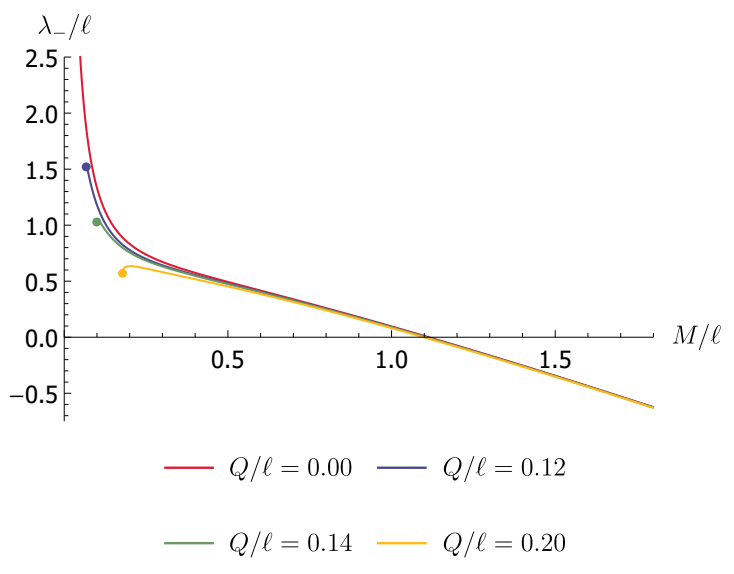

(b)

Figure 1. The thermodynamic length of the south pole is plotted as a function of the renormalized thermodynamic mass $M$ for (a) the varying tension with a fixed $Q / \ell=0.10$, and (b) the varying $Q / \ell$ with a fixed tension $\mu_{-}=0.10$. All the solid circle points represent the corresponding extremal black holes.

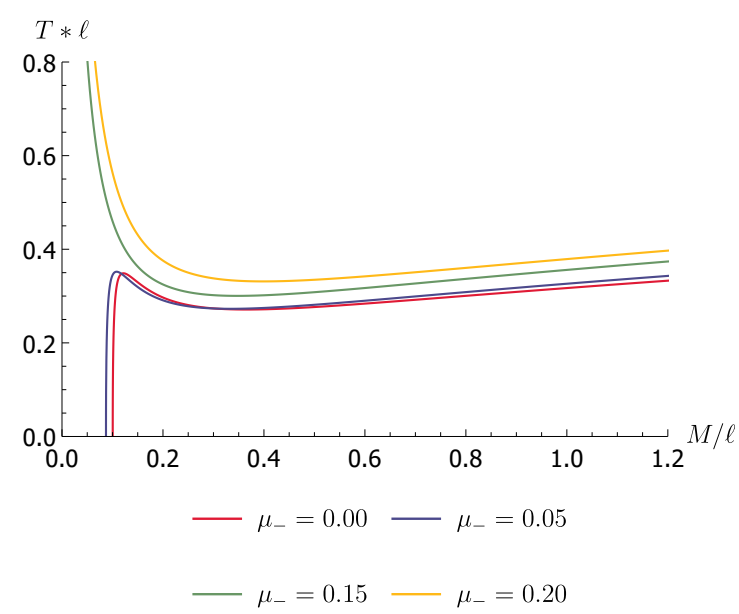

(a)

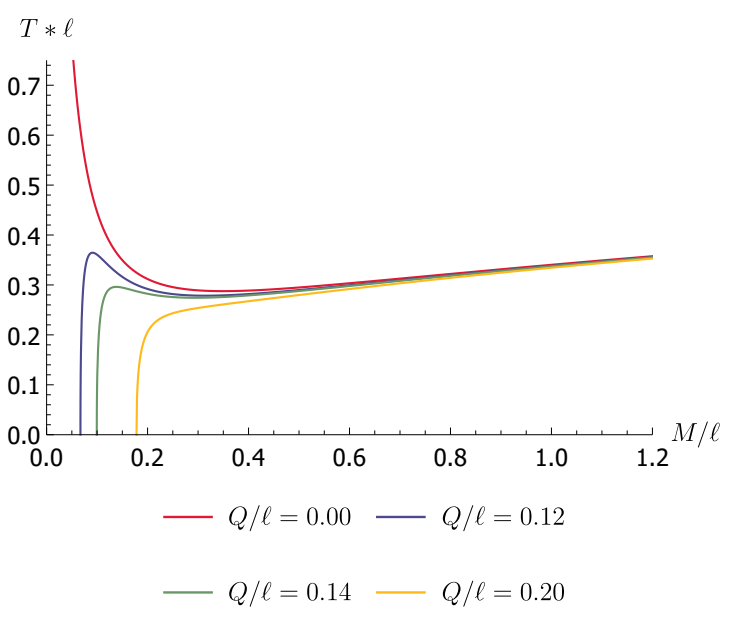

(b)

Figure 2. A plot of the temperature as a function of the thermodynamic mass for varying string tension $\mu_{-}$with fixed $Q / \ell=0.10$ in (a), and for varying $Q / \ell$ with fixed tension $\mu_{-}=0.10$ in (b).

black holes to make sense. Neither does the red curve $(Q / \ell=0)$ in figure $1(\mathrm{~b})$ with $M / \ell \gtrsim 0.0123$ (or equivalently $A \ell \lesssim 0.9925$ ).

A representative plot of the temperature as a function of the mass, $M$, for the accelerating charged black hole is illustrated in figure 2. As we can see from figure 2(a), if the string tension $\mu_{-}$is small, the temperature can approach zero as the mass decreases, signaling an extremal black hole. However, as $\mu_{-}$increases, there seems to be a critical value of $\mu_{-}$above which the extremality never occurs and the corresponding temperature has a local positive minimum value. It is difficult to obtain an analytic expression of the critical point. However, numerical calculations indicate that the value is approximately 0.10 with 


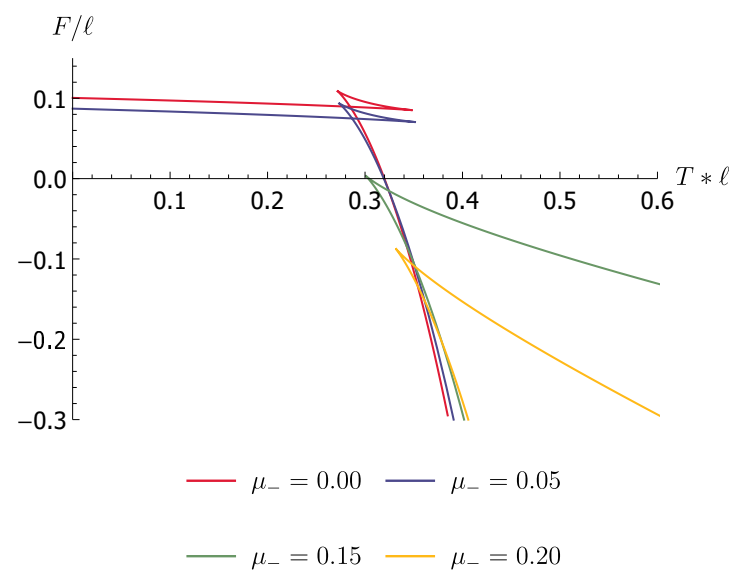

(a)

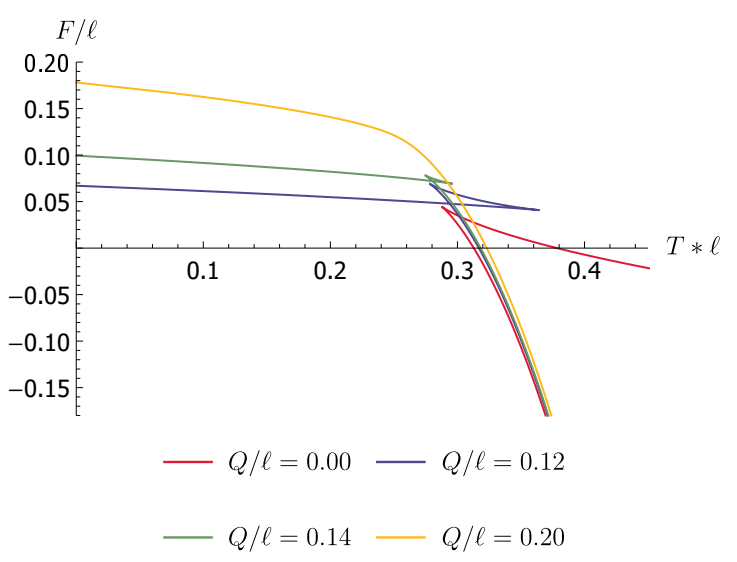

(b)

Figure 3. The free energy is plotted as a function of temperature for varying string tension $\mu_{-}$ with fixed $Q / \ell=0.10$ in (a), and varying $Q / \ell$ with fixed $\mu_{-}=0.10$ in (b).

very high precision, which happens to be the value of $Q / \ell$. In this situation, it should be emphasized that $M / \ell$ cannot go arbitrarily small in order to avoid the occurrence of the accelerating horizon. For example, for the case of $\mu_{-}=0.15, M / \ell \gtrsim 0.0232$ is required (or equivalently $A \ell \lesssim 0.9783$ ) as aforesaid, while for $\mu_{-}=0.20, M / \ell \gtrsim 0.0536$ (or equivalently $A \ell \lesssim 0.9534)$. Similarly, for a varying $Q / \ell$, as shown in figure $2(\mathrm{~b})$, the increasing $Q / \ell$ will make the charged accelerating black hole approach the extremal limit at a small $M / \ell$. If $Q / \ell$ is large enough, then temperature becomes a monotonic function of the mass and the extremality no longer happens at large enough $M / \ell$.

In figure 3, we have displayed the variation of the free energy $F=M-T S$ with the temperature for varying tension and charge in the canonical ensemble. For the case of a fixed $Q / \ell=0.10$, as the tension $\mu_{-}$is increased, the swallowtail style will be suppressed, leaving only two wings, which means that the increasing tension renders the charged accelerating black holes far away from extremality, and a spontaneous phase transition from a small black hole to a large black hole will occur. For a fixed tension, the prominent swallowtail exists in the region where $Q / \ell$ becomes comparable to $\mu_{-}$. As $Q / \ell$ is gradually increased or decreased, the swallowtail will disappear. Especially for large enough $Q / \ell$, only a single branch is left at last, which means that there is a critical point for $Q / \ell$ at which the intermediate regime with negative specific heat for the charged accelerating black hole just disappears.

\section{Benchmarking black hole heat engines and efficiency}

With the first law of charged accelerating AdS black holes derived, now we turn our attention to the features of the corresponding holographic heat engine in the benchmarking scheme. It is well-known that the efficiency for a valid heat cycle is defined by

$$
\eta=1-\frac{Q_{C}}{Q_{H}},
$$


where $Q_{C}$ denotes a net output heat flow in one cycle, and $Q_{H}$ represents a net input heat flow. In the benchmarking scheme [35, 45], a circular or elliptical cycle has been suggested to allow for cross-comparison of the efficiencies of holographic heat engines with different black holes as the working substances. In the $P-V$ plane, a general circle cycle can be written as

$$
P(\theta)=P_{0}+R \sin \theta, V(\theta)=V_{0}+R \cos \theta,
$$

where the circle is centered at $\left(V_{0}, P_{0}\right)$ with a radius of $R$. Note that the relation $R<P_{0}$ and $R<V_{0}$ should hold for a physical heat cycle.

As we have shown in the proceeding section, all the thermodynamic variables in the first law are certain combinations of the black hole parameters and the specific heat capacity at constant volume is nonzero (i.e., $C_{V} \neq 0$ ). Therefore, it is difficult to obtain the analytic expression for the efficiency of benchmarking black hole heat engines and a numerical integration of $T d S$ will be needed.

In order to better understand how the holographic heat engine of the accelerating charged black holes behaves, let us first review the influence of a charge on the temperature of the black holes. According to eq. (2.9), it is easy to see that the contribution of a charge to the temperature is negative. Therefore, there exists an upper-bound of $Q$ for the black hole thermodynamics. If $Q$ approaches the upper-bound, the temperature becomes zero and the black hole becomes extremal. Since a valid cycle contour is forbidden to cross the area of non-positive temperature, a zero isothermal curve cannot be tangent to or intersecting with the circular cycle in the $P-V$ plane.

For a benchmarking circle in the $P-V$ plane, there must be a point of minim temperature on the circle. If we suppose an extremal black hole at the point of minim temperature on the cycle, then the corresponding value of $Q$ of the extremal black hole can be considered as an extremal upper-bound of charge for the benchmarking circle. This upper-bound is denoted by $Q_{\max }$ in our following discussions. It is not difficult to deduce that if the thermodynamic charge is larger than $Q_{\max }$, negative temperature occurs and the cycle process stops. Therefore, a benchmarking heat engine of black holes with charge can make physical sense only when $Q<Q_{\max }$. In figure 4 , we have displayed how $Q_{\max }$ changes as a function of $\mu_{-}$and $R$. We can see that the upper-bound $Q_{\max }$ is a decreasing function of $\mu_{-}$and $R$. Here, it should be emphasized that the symbol $Q_{\max }$ or $Q$ in the following plots is not in units of $\ell$ but in that of charge.

Let us return to the efficiency in the benchmarking circular cycle in eq. (3.2), which will be influenced by the thermodynamic variables $Q, \mu_{-}$and the circle radius $R$. We first present the behavior of the efficiency with the increasing circle radius $R$ for fixed $Q$ and $\mu_{-}$in figure 5 . Here, the Carnot efficiency is classically defined by $\eta_{C}=1-T_{C} / T_{H}$ with maximum temperature $T_{H}$ and minim temperature $T_{C}$ in the entire cycle process, and $\eta_{D}=2 \pi /(\pi+4)$ is proposed as the universal upper-bound for the efficiencies of benchmarking holographic heat engines on circular cycles in ref. [45]. As we can see, the efficiency of the benchmarking black hole heat engine $\eta$ increases with the increase of $R$, and the corresponding Carnot efficiency $\eta_{C}$ is always larger than the efficiency $\eta$. Besides these, $\eta$ can approximate to the upper-bound $\eta_{D}$ so long as $R$ draws near 11.7955. A comment is 


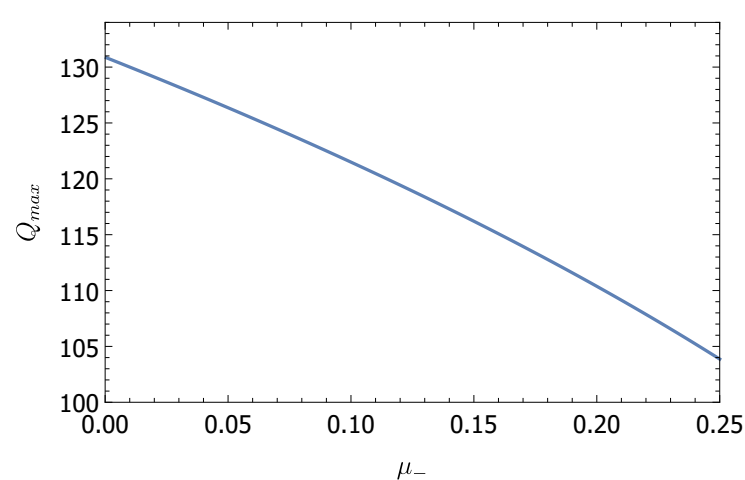

(a)

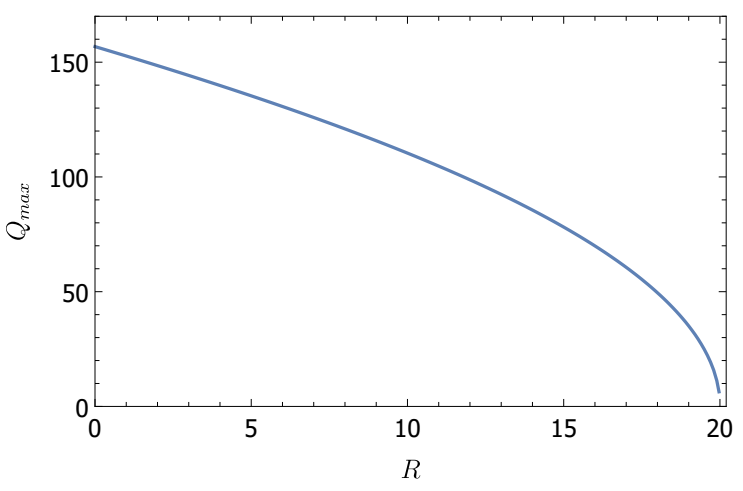

(b)

Figure 4. Assuming the benchmarking circle is at $\left(V_{0}, P_{0}\right)=(100,20)$ in the $P-V$ plane, the upper-bound of charge $Q_{\max }$ is plotted as a function of (a) $\mu_{-}$for fixed $R=10$ and (b) $R$ for fixed $\mu_{-}=0.20$.

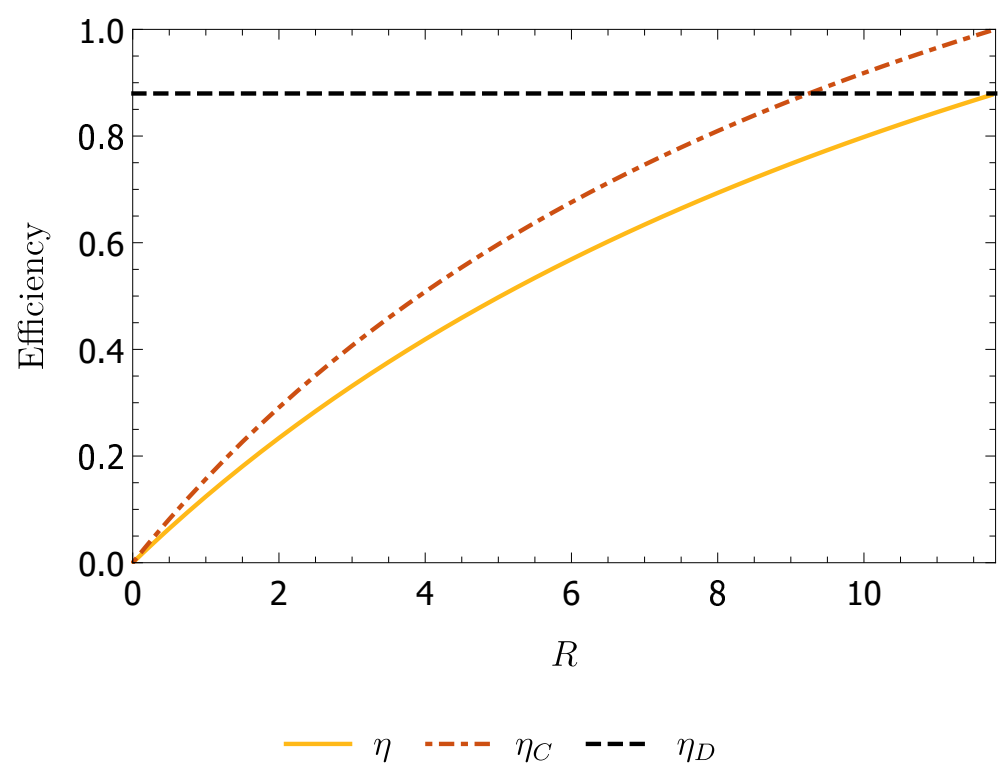

Figure 5. The efficiency of a heat engine of charged accelerating black holes is plotted as a function of $R$ with fixed $Q=100$ and $\mu_{-}=0.20$, where $\eta_{C}$ denotes Carnot efficiency and $\eta_{D}=2 \pi /(\pi+4)$ means the upper-bound value of efficiency. We have assumed the centre of the cycle satisfies $\left(V_{0}, P_{0}\right)=(100,20)$. Here, for a valid heat engine cycle, $R$ should be smaller than 11.7955 since the upper-bond $Q_{\max }=100$ for $R \approx 11.7955$ and $\mu_{-}=0.20$.

in order here. According to eq. (2.9) and eq. (3.2), it is not difficult to derive that $Q_{\max }$, the upper-bound of charge, will approximate to 100 for $\mu_{-}=0.20$ and $R=11.7955$. If $R$ goes beyond 11.7955, the $Q_{\max }$ will become smaller than 100 (see figure 4(b)), then the circular cycle for $Q=100$ will cross the region of negative temperature, which makes no sense in physics. Therefore, in the setting of $Q=100, \mu_{-}=0.20$ and $\left(V_{0}, P_{0}\right)=(100,20), R$ cannot go beyond 11.7955, and the efficiency will approximate to the upper-bound $2 \pi /(\pi+4)$ in the limit of $R$ approaching 11.7955 . 


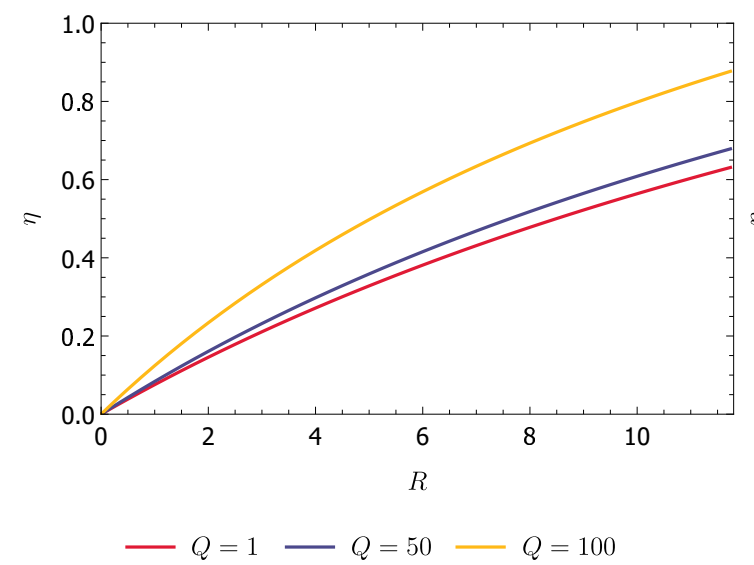

(a)

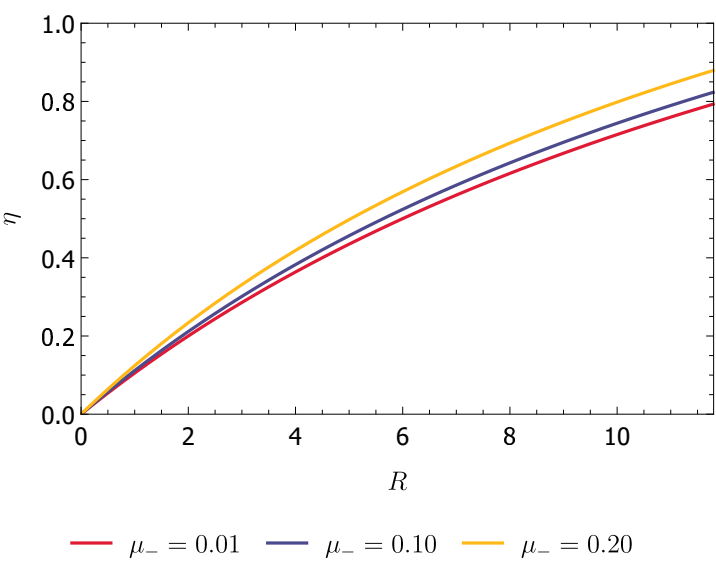

(b)

Figure 6. The efficiency of the benchmarking heat engine as a function of the radius $R$ for the circular cycle is centered at $\left(V_{0}, P_{0}\right)=(100,20)$. (a)Thermodynamic charge $Q=\{1,50,100\}$ with fixed cosmic string tension $\mu_{-}=0.20$. (b) The tension $\mu_{-}=\{0.01,0.10,0.20\}$ with fixed $Q=100$.

In figure 6 , the efficiencies are plotted as a function of the circle radius $R$ for fixed cosmic string tension and fixed charge respectively. It is easy to find that the efficiency is still an increasing function of $R$. For a fixed $R$, the larger the $Q$ or the larger the $\mu_{-}$, the higher the efficiency.

In figure 7, we give a representative plot of the efficiency as a function of the cosmic string tension $\mu_{-}$for some different values of charge $Q$, which illustrates how the variation of charge influences the curved contour describing the behavior of the efficiency with increasing $\mu_{-}$for a fixed benchmarking circle. One can see that when the value of charge $Q$ is small a variation of $\mu_{-}$does not cause significant changes in the efficiency as one would expect. However, when the value of $Q$ is large, the efficiency becomes an obvious increasing function of the cosmic string tension $\mu_{-}$in contrast to the uncharged case [46]. Therefore, the presence of a charge may significantly increase the efficiency of the benchmarking heat engine with slowly accelerating AdS black holes as working substances.

Now, we cross-compare the efficiency of different black hole heat engines in the benchmarking scheme. The black hole heat engines we choose are those of the slowly accelerating charged AdS black holes we just studied, the Einstein-Maxwell-AdS black holes (ReissnerNordstrom-like black hole) and the Born-Infeld black holes. We plot the efficiency of the benchmarking heat engine as a function of charge $Q$ with different black holes as working substances in figure 8. It is worth noting that both Einstein-Maxwell-AdS black hole and Born-Infeld black hole have a vanishing specific heat at constant volume(i.e., $C_{V}=0$, the detailed thermodynamic quantities can be found in refs. [35, 65-67]), and the holographic heat engine efficiency has been studied in refs. [31, 45].

Figure 8 reveals that the existence of charge in general increases the work efficiency to some extent. More interestingly, one finds that with acceleration, the holographic heat engine efficiency is usually larger than that without, and moreover it increases more rapidly as the charge grows. 


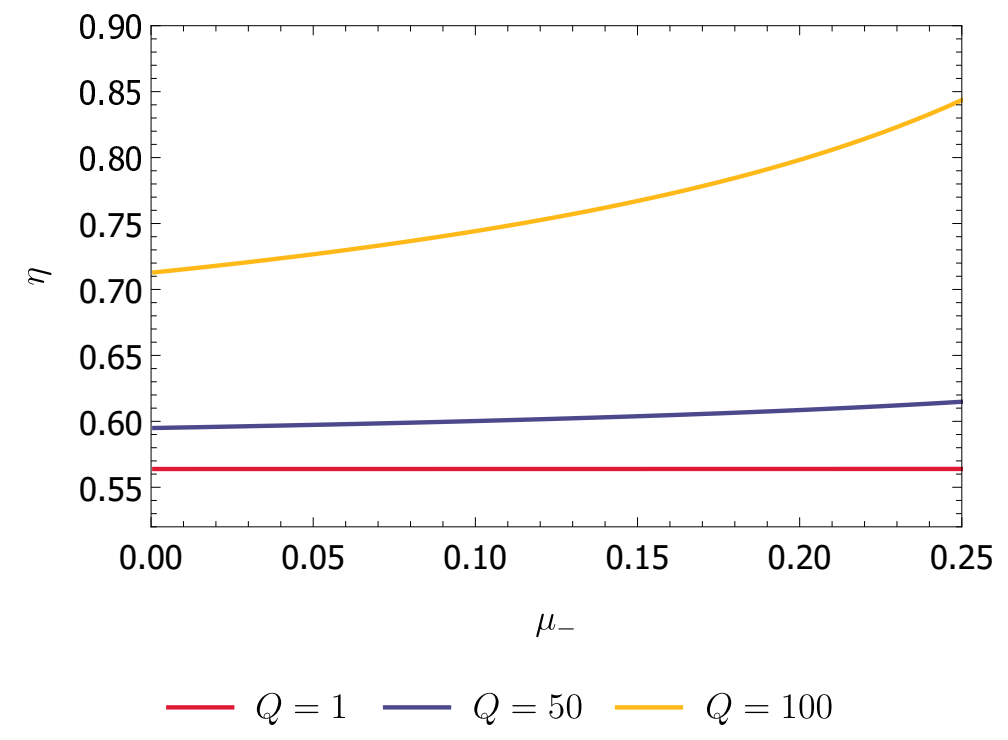

Figure 7. The efficiency of the holographic heat engine as a function of cosmic string tension $\mu_{-}$ with $Q=\{1,50,100\}$. The center of the circle is at $\left(V_{0}, P_{0}\right)=(100,20)$ with a fixed radius $R=10$.

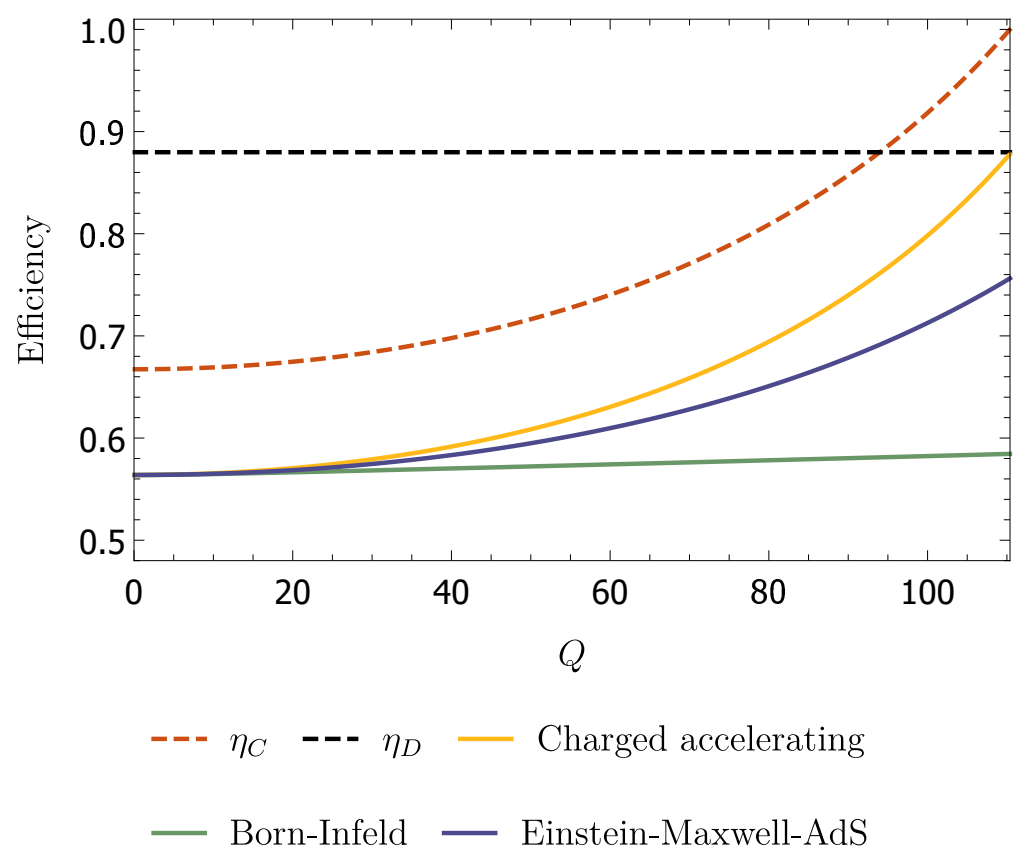

Figure 8. The efficiencies vs electric charge $Q$ with the center of benchmarking cycle localized at $\left(V_{0}, P_{0}\right)=(100,20)$ and $R=10$. The three solid curves respectively represent the efficiencies of three different families of black holes as the working material, including charged accelerating AdS $\left(\mu_{-}=0.20\right)$, Born-Infeld $(\beta=1)$ and Einstein-Maxwell-AdS black holes. Note that the extreme charge for charged accelerating AdS black holes in this benchmarking cycle reads $Q_{\max } \approx 110.3813$, of which the efficiency will draw near $\eta_{D}=2 \pi /(4+\pi)$. Here, $\eta_{C}$ denotes the Carnot efficiency of charged accelerating black hole in this circular cycle. 


\section{Conclusion}

We have explored the thermodynamics of charged accelerating AdS black holes described by $C$-metric. In order to obtain the consistent thermodynamic depiction for the charged accelerating black holes that have an uncommon asymptotical behaviour, the normalization of the timelike Killing vector is fixed by holographic methods, i.e.,

$$
\tau=\sqrt{\left(1+A^{2} e^{2}\right)\left(1-A^{2} \ell^{2}-A^{4} \ell^{2} e^{2}\right)} t
$$

and such a choice makes all the corresponding thermodynamic parameters be analogous to those of an observer comoving with the accelerating charged object. Through some algebraic manipulations, we find that the exact expression of the thermodynamic mass in the consistent first law is complicatedly intertwined with the parameters $e, A, \ell$, so do the other thermodynamic quantities, such as, the volume and the thermodynamic length. With the first law, the properties of the thermodynamic length and the possible phase behaviour of the charged accelerating black holes are also discussed in a standard thermodynamic analysis. We find that the increasing tension renders the charged accelerating black hole away from extremality, the phase transition to large black holes occurs, while increasing the charge, the intermediate regime with negative specific heat will gradually disappear.

Moreover, we have examined the features of the heat engines with charged accelerating black holes as the working substances in the benchmarking scheme of holographic heat engines. Due to the fact that the corresponding thermodynamic variables in the first law are non-linear combinations of the black hole parameters $r_{+}, m, e, \ell$ and $A$, the numerical estimation is used to demonstrate the behavior of the efficiency of the holographic heat engines. We found that the efficiency of the holographic heat engine can be influenced by the cosmic string tension, the size of circular cycle and the thermodynamic charge. When the cosmic string tension is fixed, the efficiency of the holographic heat engine is increased by the existence of charge. For a fixed size of the circular cycle, the existence of charge may significantly increase the efficiency of the holographic heat engine as the string tension grows. A cross-comparison of the holographic heat engines with slowly accelerating charged AdS black holes and the Einstein-Maxwell-AdS black holes in a fixed benchmarking cycle shows that the presence of acceleration also increases the efficiency, and moreover, it makes the efficiency more sensitive to a varying charge $Q$.

\section{Acknowledgments}

This work was supported by the National Natural Science Foundation of China under Grants No. 11435006 and No.11690034.

Open Access. This article is distributed under the terms of the Creative Commons Attribution License (CC-BY 4.0), which permits any use, distribution and reproduction in any medium, provided the original author(s) and source are credited. 


\section{References}

[1] S. Hawking, Particle creation by black holes, Commun. Math. Phys. 43 (1975) 199 [Erratum ibid. 46 (1976) 206].

[2] S. Hawking, Black holes and thermodynamics, Phys. Rev. D 13 (1976) 191.

[3] T. Jacobson, Thermodynamics of space-time: The Einstein equation of state, Phys. Rev. Lett. 75 (1995) 1260 [gr-qc/9504004] [INSPIRE].

[4] T. Padmanabhan, Thermodynamical Aspects of Gravity: New insights, Rept. Prog. Phys. 73 (2010) 046901 [arXiv:0911.5004] [InSPIRE].

[5] P. Kovtun, D.T. Son and A.O. Starinets, Viscosity in strongly interacting quantum field theories from black hole physics, Phys. Rev. Lett. 94 (2005) 111601 [hep-th/0405231] [INSPIRE].

[6] S.A. Hartnoll, P.K. Kovtun, M. Muller and S. Sachdev, Theory of the Nernst effect near quantum phase transitions in condensed matter and in dyonic black holes, Phys. Rev. B 76 (2007) 144502 [arXiv:0706.3215] [InSPIRE].

[7] S.A. Hartnoll, C.P. Herzog and G.T. Horowitz, Building a Holographic Superconductor, Phys. Rev. Lett. 101 (2008) 031601 [arXiv:0803.3295] [INSPIRE].

[8] J.E. Aman, I. Bengtsson and N. Pidokrajt, Geometry of black hole thermodynamics, Gen. Rel. Grav. 35 (2003) 1733 [gr-qc/0304015] [INSPIRE].

[9] H. Quevedo, Geometrothermodynamics of black holes, Gen. Rel. Grav. 40 (2008) 971 [arXiv:0704.3102] [INSPIRE].

[10] G. Ruppeiner, Thermodynamic curvature and black holes, Springer Proc. Phys. 153 (2014) 179 [arXiv:1309.0901] [INSPIRE].

[11] S.A.H. Mansoori and B. Mirza, Correspondence of phase transition points and singularities of thermodynamic geometry of black holes, Eur. Phys. J. C 74 (2014) 2681 [arXiv: 1308.1543] [INSPIRE].

[12] J. Suresh, R. Tharanath, N. Varghese and V.C. Kuriakose, The thermodynamics and thermodynamic geometry of the Park black hole, Eur. Phys. J. C 74 (2014) 2819 [arXiv: 1403.4710] [INSPIRE].

[13] J.-L. Zhang, R.-G. Cai and H. Yu, Phase transition and thermodynamical geometry for Schwarzschild AdS black hole in $A d S_{5} \times S^{5}$ spacetime, JHEP 02 (2015) 143 [arXiv: 1409.5305] [INSPIRE].

[14] J.-L. Zhang, R.-G. Cai and H. Yu, Phase transition and thermodynamical geometry of Reissner-Nordström-AdS black holes in extended phase space, Phys. Rev. D 91 (2015) 044028 [arXiv: 1502.01428] [InSPIRE].

[15] S.H. Hendi, A. Sheykhi, S. Panahiyan and B. Eslam Panah, Phase transition and thermodynamic geometry of Einstein-Maxwell-dilaton black holes, Phys. Rev. D 92 (2015) 064028 [arXiv: 1509.08593] [InSPIRE].

[16] C. Gruber, O. Luongo and H. Quevedo, Geometric approaches to the thermodynamics of black holes, in Proceedings of 14 th Marcel Grossmann Meeting on Recent Developments in Theoretical and Experimental General Relativity, Astrophysics and Relativistic Field Theories (MG14), Rome Italy (2015), vol. 1, pg. 453 [arXiv:1603.09443] [INSPIRE]. 
[17] R. Banerjee, B.R. Majhi and S. Samanta, Thermogeometric phase transition in a unified framework, Phys. Lett. B 767 (2017) 25 [arXiv:1611.06701] [INSPIRE].

[18] D. Kubiznak and R.B. Mann, Black hole chemistry, Can. J. Phys. 93 (2015) 999 [arXiv: 1404.2126] [INSPIRE].

[19] R.B. Mann, The Chemistry of Black Holes, Springer Proc. Phys. 170 (2016) 197 [inSPIRE].

[20] C. Teitelboim, The cosmological constant as a thermodynamic black hole parameter, Phys. Lett. B 158 (1985) 293 [INSPIRE].

[21] M.M. Caldarelli, G. Cognola and D. Klemm, Thermodynamics of Kerr-Newman-AdS black holes and conformal field theories, Class. Quant. Grav. 17 (2000) 399 [hep-th/9908022] [INSPIRE].

[22] D. Kastor, S. Ray and J. Traschen, Enthalpy and the Mechanics of AdS Black Holes, Class. Quant. Grav. 26 (2009) 195011 [arXiv:0904.2765] [INSPIRE].

[23] B.P. Dolan, The cosmological constant and the black hole equation of state, Class. Quant. Grav. 28 (2011) 125020 [arXiv: 1008.5023] [INSPIRE].

[24] B.P. Dolan, Pressure and volume in the first law of black hole thermodynamics, Class. Quant. Grav. 28 (2011) 235017 [arXiv:1106.6260] [INSPIRE].

[25] M. Cvetič, G.W. Gibbons, D. Kubiznak and C.N. Pope, Black Hole Enthalpy and an Entropy Inequality for the Thermodynamic Volume, Phys. Rev. D 84 (2011) 024037 [arXiv: 1012.2888] [INSPIRE].

[26] D. Kubiznak, R.B. Mann and M. Teo, Black hole chemistry: thermodynamics with Lambda, Class. Quant. Grav. 34 (2017) 063001 [arXiv:1608.06147] [INSPIRE].

[27] D. Kubiznak and R.B. Mann, P-V criticality of charged AdS black holes, JHEP 07 (2012) 033 [arXiv:1205.0559] [INSPIRE].

[28] N. Altamirano, D. Kubiznak and R.B. Mann, Reentrant phase transitions in rotating anti-de Sitter black holes, Phys. Rev. D 88 (2013) 101502 [arXiv:1306.5756] [INSPIRE].

[29] R.A. Hennigar, R.B. Mann and E. Tjoa, Superfluid Black Holes, Phys. Rev. Lett. 118 (2017) 021301 [arXiv: 1609.02564] [INSPIRE].

[30] C.V. Johnson, Holographic Heat Engines, Class. Quant. Grav. 31 (2014) 205002 [arXiv: 1404.5982] [INSPIRE].

[31] A. Chakraborty and C.V. Johnson, Benchmarking Black Hole Heat Engines, Int. J. Mod. Phys. D 28 (2019) 1950012 [arXiv:1612.09272] [INSPIRE].

[32] A. Belhaj, M. Chabab, H. El Moumni, K. Masmar, M.B. Sedra and A. Segui, On Heat Properties of AdS Black Holes in Higher Dimensions, JHEP 05 (2015) 149 [arXiv: 1503.07308] [INSPIRE].

[33] E. Caceres, P.H. Nguyen and J.F. Pedraza, Holographic entanglement entropy and the extended phase structure of STU black holes, JHEP 09 (2015) 184 [arXiv:1507.06069] [INSPIRE].

[34] M.R. Setare and H. Adami, Polytropic black hole as a heat engine, Gen. Rel. Grav. 47 (2015) 133 [INSPIRE].

[35] C.V. Johnson, Gauss-Bonnet black holes and holographic heat engines beyond large N, Class. Quant. Grav. 33 (2016) 215009 [arXiv:1511.08782] [INSPIRE]. 
[36] C.V. Johnson, Born-Infeld AdS black holes as heat engines, Class. Quant. Grav. 33 (2016) 135001 [arXiv:1512.01746] [INSPIRE].

[37] M. Zhang and W.-B. Liu, $f(R)$ Black Holes as Heat Engines, Int. J. Theor. Phys. 55 (2016) 5136 [inSPIRE].

[38] J. Sadeghi and K. Jafarzade, The Thermodynamic Efficiency in Static and Dynamic Black Holes, Int. J. Theor. Phys. 56 (2017) 3387 [arXiv:1504.07744] [INSPIRE].

[39] S.-W. Wei and Y.-X. Liu, Charged AdS black hole heat engines, arXiv:1708.08176 [INSPIRE].

[40] S.H. Hendi, B. Eslam Panah, S. Panahiyan, H. Liu and X.H. Meng, Black holes in massive gravity as heat engines, Phys. Lett. B 781 (2018) 40 [arXiv:1707.02231] [INSPIRE].

[41] C. Bhamidipati and P.K. Yerra, A note on Gauss-Bonnet black holes at criticality, Phys. Lett. B 772 (2017) 800 [arXiv: 1706.09344] [INSPIRE].

[42] H. Xu, Y. Sun and L. Zhao, Black hole thermodynamics and heat engines in conformal gravity, Int. J. Mod. Phys. D 26 (2017) 1750151 [arXiv:1706.06442] [INSPIRE].

[43] H. Liu and X.-H. Meng, Effects of dark energy on the efficiency of charged AdS black holes as heat engines, Eur. Phys. J. C 77 (2017) 556 [arXiv: 1704.04363] [InSPIRE].

[44] J.-X. Mo, F. Liang and G.-Q. Li, Heat engine in the three-dimensional spacetime, JHEP 03 (2017) 010 [arXiv: 1701.00883] [INSPIRE].

[45] R.A. Hennigar, F. McCarthy, A. Ballon and R.B. Mann, Holographic heat engines: general considerations and rotating black holes, Class. Quant. Grav. 34 (2017) 175005 [arXiv: 1704.02314] [INSPIRE].

[46] J. Zhang, Y. Li and H. Yu, Accelerating AdS black holes as the holographic heat engines in a benchmarking scheme, Eur. Phys. J. C 78 (2018) 645 [arXiv:1801.06811] [INSPIRE].

[47] B. Eslam Panah, Effects of energy dependent spacetime on geometrical thermodynamics and heat engine of black holes: gravity's rainbow, Phys. Lett. B 787 (2018) 45 [arXiv: 1805.03014] [INSPIRE].

[48] W. Kinnersley and M. Walker, Uniformly accelerating charged mass in general relativity, Phys. Rev. D 2 (1970) 1359 [INSPIRE].

[49] J.F. Plebanski and M. Demianski, Rotating, charged and uniformly accelerating mass in general relativity, Annals Phys. 98 (1976) 98 [INSPIRE].

[50] O.J.C. Dias and J.P.S. Lemos, Pair of accelerated black holes in anti-de Sitter background: AdS C metric, Phys. Rev. D 67 (2003) 064001 [hep-th/0210065] [INSPIRE].

[51] J.B. Griffiths and J. Podolsky, A New look at the Plebanski-Demianski family of solutions, Int. J. Mod. Phys. D 15 (2006) 335 [gr-qc/0511091] [InSPIRE].

[52] F. Dowker, J.P. Gauntlett, D.A. Kastor and J.H. Traschen, Pair creation of dilaton black holes, Phys. Rev. D 49 (1994) 2909 [hep-th/9309075] [INSPIRE].

[53] R. Emparan and H.S. Reall, A Rotating black ring solution in five-dimensions, Phys. Rev. Lett. 88 (2002) 101101 [hep-th/0110260] [INSPIRE].

[54] J. Podolsky, M. Ortaggio and P. Krtous, Radiation from accelerated black holes in an anti-de Sitter universe, Phys. Rev. D 68 (2003) 124004 [gr-qc/0307108] [INSPIRE]. 
[55] M. Appels, R. Gregory and D. Kubiznak, Thermodynamics of Accelerating Black Holes, Phys. Rev. Lett. 117 (2016) 131303 [arXiv: 1604.08812] [INSPIRE].

[56] M. Appels, R. Gregory and D. Kubiznak, Black Hole Thermodynamics with Conical Defects, JHEP 05 (2017) 116 [arXiv: 1702.00490] [INSPIRE].

[57] R. Gregory, Accelerating Black Holes, J. Phys. Conf. Ser. 942 (2017) 012002 [arXiv: 1712.04992] [INSPIRE].

[58] M. Astorino, Thermodynamics of Regular Accelerating Black Holes, Phys. Rev. D 95 (2017) 064007 [arXiv: 1612.04387] [INSPIRE].

[59] A. Anabalón, M. Appels, R. Gregory, D. Kubizňák, R.B. Mann and A. Ovgün, Holographic Thermodynamics of Accelerating Black Holes, Phys. Rev. D 98 (2018) 104038 [arXiv: 1805.02687] [INSPIRE].

[60] M. Appels, Thermodynamics of Accelerating Black Holes, Ph.D. Thesis, Durham University, Durham U.K. (2018).

[61] A. Anabalón, F. Gray, R. Gregory, D. Kubizňák and R.B. Mann, Thermodynamics of Charged, Rotating and Accelerating Black Holes, arXiv:1811.04936 [INSPIRE].

[62] G.W. Gibbons, M.J. Perry and C.N. Pope, The First law of thermodynamics for Kerr-anti-de Sitter black holes, Class. Quant. Grav. 22 (2005) 1503 [hep-th/0408217] [INSPIRE].

[63] A. Ashtekar and S. Das, Asymptotically Anti-de Sitter space-times: Conserved quantities, Class. Quant. Grav. 17 (2000) L17 [hep-th/9911230] [INSPIRE].

[64] S. Das and R.B. Mann, Conserved quantities in Kerr-anti-de Sitter space-times in various dimensions, JHEP 08 (2000) 033 [hep-th/0008028] [INSPIRE].

[65] A. Chamblin, R. Emparan, C.V. Johnson and R.C. Myers, Charged AdS black holes and catastrophic holography, Phys. Rev. D 60 (1999) 064018 [hep-th/9902170] [InSPIRE].

[66] R.-G. Cai, D.-W. Pang and A. Wang, Born-Infeld black holes in (A)dS spaces, Phys. Rev. D 70 (2004) 124034 [hep-th/0410158] [INSPIRE].

[67] T.K. Dey, Born-Infeld black holes in the presence of a cosmological constant, Phys. Lett. B 595 (2004) 484 [hep-th/0406169] [INSPIRE]. 